\title{
Cancer exosomes in urine: a promising biomarker source
}

\author{
Isabella Panfoli \\ Dipartimento di Farmacia-DIFAR, Laboratorio di Biochimica, Università di Genova, Genoa, Italy \\ Correspondence to: Prof. Isabella Panfoli. Biochemistry Laboratory, DIFAR-University of Genoa, Viale Benedetto XV 3, Genova 16132, Italy. \\ Email: panfoli@difar.unige.it.
}

\begin{abstract}
In face of innovative therapeutic strategies for cancer diagnosis and treatment, and of the elusiveness of renal and urinary tract tumors, the identification of novel biomarkers for diagnosis, prognosis, selection and monitoring of therapies is a primary target of research. The concept of "precision medicine" for tailoring the oncologic management in a minimally invasive fashion is considered a new epoch in cancer management. Extracellular vesicles, and exosomes in particular, carry lipids, mRNAs, non-coding RNAs, DNA, and active proteins, are present in a variety of bodily fluids including urine. In fact, urinary exosomes contain most of the urinoma proteins. Exosomes are nanovesicles originating from an endocytic pathway of the endocellular membranes and upon release are actors of intercellular communication, able to induce phenotypic changes including tumorigenesis and metastasis, in recipient cells. Tumor-derived exosomes play critical roles in all stages of tumor development and metastasis of almost all cancer types. Exosomal proteins may serve as biomarkers for clinical applications, still to be validated. Please reword the next sentence. Not clear. Exosomes extracellular gather in urine, thus becoming a great resource for recovery of biomarkers and a promising non-invasive diagnostic instrument for renal disease. Liquid biopsies (body fluids) may be preferable to tumor tissue biopsies since they are less invasive. Urinary exosomes in particular are available in great quantity in a noninvasive way and are representative of each of the cells of the urinary tract. In the present review, we summarize our knowledge of the urine exosomes with a new vision as liquid biopsy and high-throughput techniques. The emerging metabolic signature of urinary exosomes is also discussed in terms of its potential clinical application.
\end{abstract}

Keywords: Aerobic metabolism; biomarkers; cancer; exosome; intercellular communication; tumor-derived exosomes; urine

Submitted Jul 06, 2017. Accepted for publication Sep 25, 2017.

doi: $10.21037 /$ tcr.2017.10.17

View this article at: http://dx.doi.org/10.21037/tcr.2017.10.17

\section{Urinary exosomes}

Exosomes in human urine would exclusively derive from each cell type of the urinary tract starting from the glomerular podocytes and the kidney (1-3). In fact, the glomerular filtration apparatus apparently prevents blood-borne vesicles from entering the nephron (4). Exosomes in the urinary tract can play a cell-signaling role. For example, exosomes released by collecting duct cells induce the expression of aquaporin in recipient cells (5) and stimulate nephrogenesis (6). Exosomes can also simply cross the whole urinary tract being ultimately found in the urine. Being cell type specific, especially the urinary exosomes are a biomarker source for cancer diagnosis
$(2,7)$ and also for diseases of the urinary tract $(8)$. The protein content of exosomes conveyed into urine is specific (9), being even representative of the expression levels (10) within the cell of origin.

The exosomal urinary proteome has been catalogued (11). The most recent version of the exosome database, Exocarta (Version 4), reports that exosomes from a variety of cell sources have been found to contain 4,563 proteins, 194 lipids, 1,639 mRNAs, and 764 micro (mi)RNAs (12). Micro RNAs are small, non-coding RNAs that posttranscriptionally inhibit gene expression and are therefore important players in exosome key functions (13). Exosomes also contain DNA and functional long non-coding RNAs 
(lncRNAs) which may induce cancer-like phenotypes (14). We previously conducted a proteomic analysis of urinary exosomes by Orbitrap mass spectrometry, and compared it to urinome $(15,16)$. It was found that most proteins in the urinome are in exosomes. Moreover, a consistent number of the identified proteins unexpectedly clustered to aerobic ATP production. Cytoscape software analysis allowed us to assess that most of their gene name signature was associated with aerobic glucose metabolism (15). In fact, similarly to human exosomes from umbilical cord MSC (17), urinary exosomes consumed oxygen to aerobically synthesize ATP (15).

\section{Exosome composition and biogenesis}

Exosomes are membrane-derived vesicles of 30-100 nm, shed from several types of cells $(12,18)$ especially malignant ones (19). Exosomes are found in consistent concentrations in all body fluids, including blood, saliva and urine (20-22). Exosomes promote intercellular exchange of molecular information in physiological and pathological processes, including inflammation and cancer (23-25). Exosomes carry a variety of proteins, DNA and RNA molecules (25) and lipids, among which glycerophospholipids, sphingolipids, and especially cholesterol (8), but also eicosanoids. The latter are involved in tumor growth but also its prevention: exosomal docosahexaenoic acid promotes dendritic cell function (26).

Exosome biogenesis and the processes involved in the selection, packaging, and release of its cargo remain undefined. The endosomal sorting complex required for transport (ESCRT) is involved in exosome formation, but a key role was found for syndecan-syntenin-ALIX (27). A regulatory role for heparan sulfate in exosome production/secretion was proposed, as overexpression of the endoglycosidase enzyme heparanase increased tumor cell exosome secretion (28). Sequence motifs present in miRNAs are involved in their sorting into exosomes (29). Exosome protein content depends on the cell of origin, being enriched with fusion proteins, heat shock proteins, membrane trafficking proteins (Rab proteins), cytoplasmic enzymes, and the cited functional redox chain complexes I to $\mathrm{V}(15,17)$. Incompletely understood is the mechanisms underlying the sorting of proteins into exosomes. This topic is the object of several studies, which unfortunately yet have not addressed the key point of how can mitochondrial proteins be part of the exosome. Considering the complex assembly of the mitochondrial redox chains (30) inside the inner mitochondrial membranes (IMMs), and absence of contact with mitochondria during the exosome biogenesis, conceivably the redox chain proteins must already part of the endosomal membrane from which exosomes bud (31). A fusion of IMM with the endoplasmic reticulum (ER) was reported (23). There is a phospholipid exchange among IMM and the ER (32) also through the mitochondria associated membranes (33). The exosome release mechanism remains elusive; it was proposed to be $\mathrm{Ca}^{2+}$-dependent, and in some tumor cells Rab GTPasedependent (25). Exosomes interact with target cells mostly by endocytosis but also plasma membrane fusion (34). It was postulated that mesenchymal stem cells (MSCs) exosomes can carry out protein complementation of biochemically active enzymes (35) to correct cellular ATP deficit $(36,37)$. It is tempting to presume that among these enzymes there are the exosomal redox chains proteins $(15,17)$.

\section{Urinary exosomes in cancer biology}

The role of exosomes in cancer biology is an active area of investigation. Compared to healthy cells, tumor cells secrete a higher amount of exosomes $(19,38)$, that are implied in tumorigenesis (39). Exosomes were shown to enhance migration, invasion and angiogenesis in bladder, prostate and renal cancer (38). Cancer cell support the cancer micro-environment of neighboring cells (40), through establishing a pre-metastatic niche $(19,41)$, assisting the tumor in immune evasion and reprogramming of fibroblasts to myofibroblasts (42). Urinary exosomes possess oncogenic properties, including the promotion of cell migration and angiogenesis, blockade of apoptosis and of immunity (38).

\section{Renal cell carcinoma (RCC)}

RCC accounts for $3 \%$ of human malignancies. If localized RCC is often curable by surgery. However, only $12 \%$ patients with metastatic RCC are alive at 5 years. In search for biomarkers that are particularly needed for this type of cancer, a comparative proteomics was conducted on urinary exosomes isolated from urines of RCC patient compared with control subjects (43). This study has shown that RCC exosome protein content is substantially and reproducibly different from control UE. Interestingly, among the proteins that are not shared between RCC and control exosomes are those involved in matrix remodeling, over-expressed in RCC patients (43). RCC also possess a population of cancer stem cells (CSCs) that secrete large amount of extracellular vesicles that correlated with the disease progression and the 
metastatic potential (44) promoting tumor cell development and vascularization. Since endothelial cells are critical for angiogenesis and tumor metastasis, their recruitment by cancer cells appears extremely interesting (44).

\section{Bladder cancer}

High-grade bladder cancer is associated to high mortality rates. The role of human bladder cancer cell-derived exosomes on tumor cells viability was addressed in a study utilizing exosomes purified from the supernatants of bladder cancer T24 cell cultures. This study demonstrated that bladder cancer cell-derived exosomes inhibited tumor cell apoptosis in bladder-cancer lines (45). Exosomes from human bladder cancer cell lines (TCC-SUP) and urine of patients with high grade bladder cancer were found to promote angiogenesis and migration of bladder cancer cells. In particular, EDIL-3 was found to facilitate angiogenesis, and urothelial and endothelial cell migration. Consistently, exosomes purified from the urine of patients with high grade bladder cancer contained significantly higher EDIL-3 levels than healthy controls (46). The evidence that bladder cancer-derived exosomes affect bladder cancer progression, allows hypothesizing that targeting excretion and survival of exosomes can lead to novel therapeutic treatment strategies for this kind of cancer.

\section{Prostate cancer}

New biomarkers are needed for the diagnosis of prostate cancer, especially for the low-risk group (47). In fact, PSA lacks specificity and sensitivity. It was reported that urine collected after prostate massage contain exosomes secreted from prostate cancer cells (48). By next generation sequencing, the miRNA expression in a cohort of prostate cancer patients versus controls was studied. It was found that miRNA isoforms (isomiR-21, -204 and -375) with 3' end modifications were highly discriminatory between control and affected men, with a high diagnostic performance. Proteins form exosomes isolated from urine after massage were analyzed by LC-MS/MS and it was found that eleven proteins were increased in patients with prostate cancer, compared to those with negative biopsy. In particular, fatty acid binding protein 5 (FABP5) appeared to be the best potential biomarker to predict high-grade (Gleason score $>7$ ) prostate cancer. Interestingly, not only exosomal proteins and miRNA have a discriminative potential in prostate cancer, but also exosomal lipids. Their content appears to be significantly different among patients and controls (24). Lipid identification in urine exosomes as prostate cancer biomarkers can be envisaged. Notably, the cargos of exosomes are associated alto to the tumor growth, metastasis and drug resistance of prostate cancer. prostate cancer cells exosomes modulate surrounding stromal cells modulating microenvironment (47). This allows to foresee that in the next future urinary exosomes may carry discriminatory information which is useful to generate stronger biomarkers.

\section{Urine as a privileged tool for liquid biopsy}

In the field of precision medicine, the development of a noninvasive "liquid biopsy" represents a significant innovation, having the potential to reduce tumor biopsies. In urologic cancers, liquid biopsy is a privileged non-invasive tool for biomarker discovery (49). Liquid biopsies can be analyzed by high-throughput technologies at the cellular, circulating cellfree DNA (cfDNA), RNA, exosome and metabolome levels as a source for tumor-derived information (49). Analysis of the urine is particularly promising for urinary tract malignancies $(2,4)$. In addition to the importance of markers of cancer in urinary exosomes, finding markers of kidney failure would also be important, as most kidney and urinary tract diseases have a late clinical presentation.

Urine is a known source for biomarkers in various urinary tract cancers, such as bladder, kidney and prostate $(4,6)$. In order to harness the potential usefulness of urinary exosomes in cancer, standardization of the isolation method among the laboratories is going to be a fundamental topic. For example, isolation can be performed by ultracentrifugation or by double-filtration microfluidics. A recent report in which exosomes were isolated from urine by an integrated doublefiltration microfluidic system found that the concentration of urinary exosomes was significantly higher in bladder cancer patients than in healthy controls (50).

With respect to blood-based biomarkers, for which a major challenge remains the possibility to contextualize peripheral markers to the complex tumor microenvironment, urine exosomes appear more focused to it, as they would come from relatively near cancer tissues moreover sharing a common embryonal origin. In this respect, urine exosomes can better allow to detect membrane proteins from the tumor. In fact, we have previously shown that most urinary proteins occur in the exosomes and these preserve them more efficiently than in total urine, a challenging fluid (15). Moreover, as the metabolic ability of the exosomes seems to 
reflect that the cell of origin (17), it can be envisaged that one more biomarker may be searched for, particularly in urine that represent an unlimited source of exosomes.

Although urine exosome analysis as a source for biomarker proteins in search for renal and urinary tract tumors still needs validation to be transferred to clinical practice, it represents the logical extension of personalized medicine intended to tailor treatment to the individual. The perspective to have powerful tool to identify reliable prognostic and predictive biomarker for the diagnosis and tailored therapy specific urinary tract cancers appears particularly promising and therefore deserves implementation.

\section{Acknowledgements}

None.

\section{Footnote}

Conflicts of Interest: The author has no conflicts of interest to declare.

\section{References}

1. Dear JW, Street JM, Bailey MA. Urinary exosomes: A reservoir for biomarker discovery and potential mediators of intrarenal signalling. Proteomics 2013;13:1572-80.

2. Gámez-Valero A, Lozano-Ramos SI, Bancu I, et al. Urinary Extracellular Vesicles as Source of Biomarkers in Kidney Diseases. Front Immunol 2015;6:6.

3. Bourderioux M, Nguyen-Khoa T, Chhuon C, et al. A new workflow for proteomic analysis of urinary exosomes and assessment in cystinuria patients. J Proteome Res 2015;14:567-77.

4. Pisitkun T, Johnstone R, Knepper MA. Discovery of urinary biomarkers. Mol Cell Proteomics 2006;5:1760-71.

5. Street JM, Birkhoff W, Menzies RI, et al. Exosomal transmission of functional aquaporin 2 in kidney cortical collecting duct cells. J Physiol 2011;589:6119-27.

6. Quandt D, Dieter Zucht H, Amann A, et al. Implementing liquid biopsies into clinical decision making for cancer immunotherapy. Oncotarget 2017;8:48507-20.

7. Moon PG, You S, Lee JE, et al. Urinary exosomes and proteomics. Mass Spectrom Rev 2011;30:1185-202.

8. Lin J, Li J, Huang B, et al. Exosomes: Novel Biomarkers for Clinical Diagnosis. ScientificWorldJournal 2015;2015:657086.

9. Dimov I, Jankovic Velickovic L, Stefanovic V. Urinary exosomes. ScientificWorldJournal 2009;9:1107-18.

10. Hogan MC, Johnson KL, Zenka RM, et al.

Subfractionation, characterization, and in-depth proteomic analysis of glomerular membrane vesicles in human urine. Kidney Int 2014;85:1225-37.

11. Conde-Vancells J, Rodriguez-Suarez E, Embade N, et al. Characterization and comprehensive proteome profiling of exosomes secreted by hepatocytes. J Proteome Res 2008;7:5157-66.

12. Mathivanan S, Ji H, Simpson RJ. Exosomes: extracellular organelles important in intercellular communication. J Proteomics 2010;73:1907-20.

13. Kosaka N, Yoshioka Y, Hagiwara K, et al. Trash or Treasure: extracellular microRNAs and cell-to-cell communication. Front Genet 2013;4:173.

14. Gezer U, Özgür E, Cetinkaya M, et al. Long non-coding RNAs with low expression levels in cells are enriched in secreted exosomes. Cell Biol Int 2014;38:1076-9.

15. Bruschi M, Santucci L, Ravera S, et al. Human urinary exosome proteome unveils its aerobic respiratory ability. J Proteomics 2016;136:25-34.

16. Bruschi M, Ravera S, Santucci L, et al. Human urinary exosome as a metabolic effector cargo. Expert Rev Proteomics 2015;12:425-32.

17. Panfoli I, Ravera S, Podestà $M$, et al. Exosomes from human mesenchymal stem cells conduct aerobic metabolism in term and preterm newborn infants. FASEB J 2016;30:1416-24.

18. Raposo G, Stoorvogel W. Extracellular vesicles: exosomes, microvesicles, and friends. J Cell Biol 2013;200:373-83.

19. Kharaziha P, Ceder S, Li Q, et al. Tumor cell-derived exosomes: a message in a bottle. Biochim Biophys Acta 2012;1826:103-11.

20. Vlassov AV, Magdaleno S, Setterquist R, et al. Exosomes: current knowledge of their composition, biological functions, and diagnostic and therapeutic potentials. Biochim Biophys Acta 2012;1820:940-8.

21. Wang D, Sun W. Urinary extracellular microvesicles: isolation methods and prospects for urinary proteome. Proteomics 2014;14:1922-32.

22. Lässer C, Alikhani VS, Ekström K, et al. Human saliva, plasma and breast milk exosomes contain RNA: uptake by macrophages. J Transl Med 2011;9:9.

23. Clayton A, Al-Taei S, Webber J, et al. Cancer exosomes express CD39 and CD73, which suppress T cells through adenosine production. J Immunol 2011;187:676-83.

24. Llorente A, Skotland T, Sylvänne T, et al. Molecular lipidomics of exosomes released by $\mathrm{PC}-3$ prostate cancer 
cells. Biochim Biophys Acta 2013;1831:1302-9.

25. H Rashed M, Bayraktar E, K Helal G, et al. Exosomes: From Garbage Bins to Promising Therapeutic Targets. Int J Mol Sci 2017;18: pii: E538.

26. Pitt JM, Charrier M, Viaud S, et al. Dendritic CellDerived Exosomes as Immunotherapies in the Fight against Cancer. J Immunol 2014;193:1006-11.

27. Baietti MF, Zhang Z, Mortier E, et al. Syndecan-synteninALIX regulates the biogenesis of exosomes. Nat Cell Biol 2012;14:677-85.

28. Thompson CA, Purushothaman A, Ramani VC, et al. Heparanase regulates secretion, composition, and function of tumor cell-derived exosomes. J Biol Chem 2013;288:10093-9.

29. Zhang J, Li S, Li L, et al. Exosome and Exosomal MicroRNA: Trafficking, Sorting, and Function. Genomics Proteomics Bioinformatics 2015;13:17-24.

30. Genova ML, Baracca A, Biondi A, et al. Is supercomplex organization of the respiratory chain required for optimal electron transfer activity? Biochim Biophys Acta 2008;1777:740-6.

31. Panfoli I, Ravera S, Bruschi M, et al. Proteomics unravels the exportability of mitochondrial respiratory chains. Expert Rev Proteomics 2011;8:231-9.

32. Lebiedzinska M, Szabadkai G, Jones AW, et al. Interactions between the endoplasmic reticulum, mitochondria, plasma membrane and other subcellular organelles. Int J Biochem Cell Biol 2009;41:1805-16.

33. Raturi A, Simmen T. Where the endoplasmic reticulum and the mitochondrion tie the knot: the mitochondriaassociated membrane (MAM). Biochim Biophys Acta 2013;1833:213-24.

34. Cocucci E, Meldolesi J. Ectosomes and exosomes: shedding the confusion between extracellular vesicles. Trends Cell Biol 2015;25:364-72.

35. Lai RC, Yeo RW, Tan KH, et al. Mesenchymal stem cell exosome ameliorates reperfusion injury through proteomic complementation. Regen Med 2013;8:197-209.

36. Arslan F, Lai RC, Smeets MB, et al. Mesenchymal stem cell-derived exosomes increase ATP levels, decrease oxidative stress and activate PI3K/Akt pathway to enhance myocardial viability and prevent adverse remodeling after myocardial ischemia/reperfusion injury. Stem Cell Res
2013;10:301-12.

37. Lai RC, Yeo RW, Lim SK. Mesenchymal stem cell exosomes. Semin Cell Dev Biol 2015;40:82-8.

38. Franzen CA, Blackwell RH, Foreman KE, et al. Urinary Exosomes: The Potential for Biomarker Utility, Intercellular Signaling and Therapeutics in Urological Malignancy. J Urol 2016;195:1331-9.

39. Zhang X, Yuan X, Shi H, et al. Exosomes in cancer: small particle, big player. J Hematol Oncol 2015;8:83.

40. Hanahan D, Weinberg RA. Hallmarks of cancer: The next generation. Cell 2011;144:646-74.

41. Rabinowits G, Gerçel-Taylor C, Day JM, et al. Exosomal microRNA: a diagnostic marker for lung cancer. Clin Lung Cancer 2009;10:42-6.

42. Maas SLN, Breakefield XO, Weaver AM. Extracellular Vesicles: Unique Intercellular Delivery Vehicles. Trends Cell Biol 2017;27:172-88.

43. Raimondo F, Morosi L, Corbetta S, et al. Differential protein profiling of renal cell carcinoma urinary exosomes. Mol Biosyst 2013;9:1220.

44. Lindoso RS, Collino F, Camussi G, et al. Extracellular vesicles derived from renal cancer stem cells induce a protumorigenic phenotype in mesenchymal stromal cells. Oncotarget 2015;6:7959-69.

45. Yang L, Wu XH, Wang D, et al. Bladder cancer cellderived exosomes inhibit tumor cell apoptosis and induce cell proliferation in vitro. Mol Med Rep 2013;8:1272-8.

46. Beckham CJ, Olsen J, Yin PN, et al. Bladder Cancer Exosomes Contain EDIL-3/Del1 and Facilitate Cancer Progression. J Urol 2014;192:583-92.

47. Pan J, Ding M, Xu K, et al. Exosomes in diagnosis and therapy of prostate cancer. Oncotarget 2015;5:1-8.

48. Zijlstra C, Stoorvogel W. Prostasomes as a source of diagnostic biomarkers for prostate cancer. J Clin Invest 2016;126:1144-51.

49. Di Meo A, Bartlett J, Cheng Y, et al. Liquid biopsy: a step forward towards precision medicine in urologic malignancies. Mol Cancer 2017;16:80.

50. Liang LG, Kong MQ, Zhou S, et al. An integrated doublefiltration microfluidic device for isolation, enrichment and quantification of urinary extracellular vesicles for detection of bladder cancer. Sci Rep 2017;7:46224.
Cite this article as: Panfoli I. Cancer exosomes in urine: a promising biomarker source. Transl Cancer Res 2017;6(Suppl 8):S1389-S1393. doi: 10.21037/tcr.2017.10.17 